\title{
Calculation of Synthetic Ionizing Spectra for Planetary Nebulae
}

\author{
Thomas Rauch \\ Institut für Astronomie und Astrophysik, Universität Tübingen, Sand 1, \\ D-72076 Tübingen, Germany
}

\begin{abstract}
We present a new grid (solar and halo abundance ratios) of state-of-the-art fully line-blanketed NLTE model atmospheres which covers the parameter range of central stars of planetary nebulae. The grid is available at the WWW.
\end{abstract}

\section{Introduction}

During their evolution, central stars of planetary nebulae (CSPN) can reach extremely high effective temperatures (more than $100 \mathrm{kK}$ ). Since NLTE effects are particularly important for hot stars, the use of reliable NLTE stellar model atmosphere fluxes is required for an adequate spectral analysis of these stars.

The precise analysis of properties of planetary nebulae is strongly dependent on the ionizing spectrum: Observations as well as NLTE model atmosphere calculations have shown that spectra of their exciting stars neither have something to do with black-body spectra nor can be modeled sufficiently well with "standard" NLTE atmosphere models which are composed out of hydrogen and helium only: Strong differences between synthetic spectra from these compared to the observed spectra at energies higher than $54 \mathrm{eV}$ (He II ground state) can be ascribed to the neglected metal-line blanketing.

For a reliable calculation of the emergent stellar flux as ionizing spectrum for planetary nebulae the consideration of opacities from all elements from hydrogen up to the iron-group is required (Armsdorfer et al. 2002).

The accelerated lambda iteration (ALI) method represents a powerful tool to calculate metal-line blanketed atmospheres with more than 300 atomic levels treated consistently in NLTE. Thus, together with recent atomic data from the Opacity Project and Kurucz's line lists, we can calculate realistic atmospheres with millions of lines included.

\section{NLTE Model Atmospheres}

Our model atmospheres are calculated using the NLTE code PRO2 (Werner 1986, Werner \& Dreizler 1999). The models are plane-parallel and in hydrostatic and radiative equilibrium. More than 300 levels can be treated in NLTE with more than 1000 line transitions considered in detail. Millions of lines of the iron-group elements tabulated in Kurucz (1996) and data from the Opacity Project (Seaton et al.1994) are accounted for using an opacity sampling 


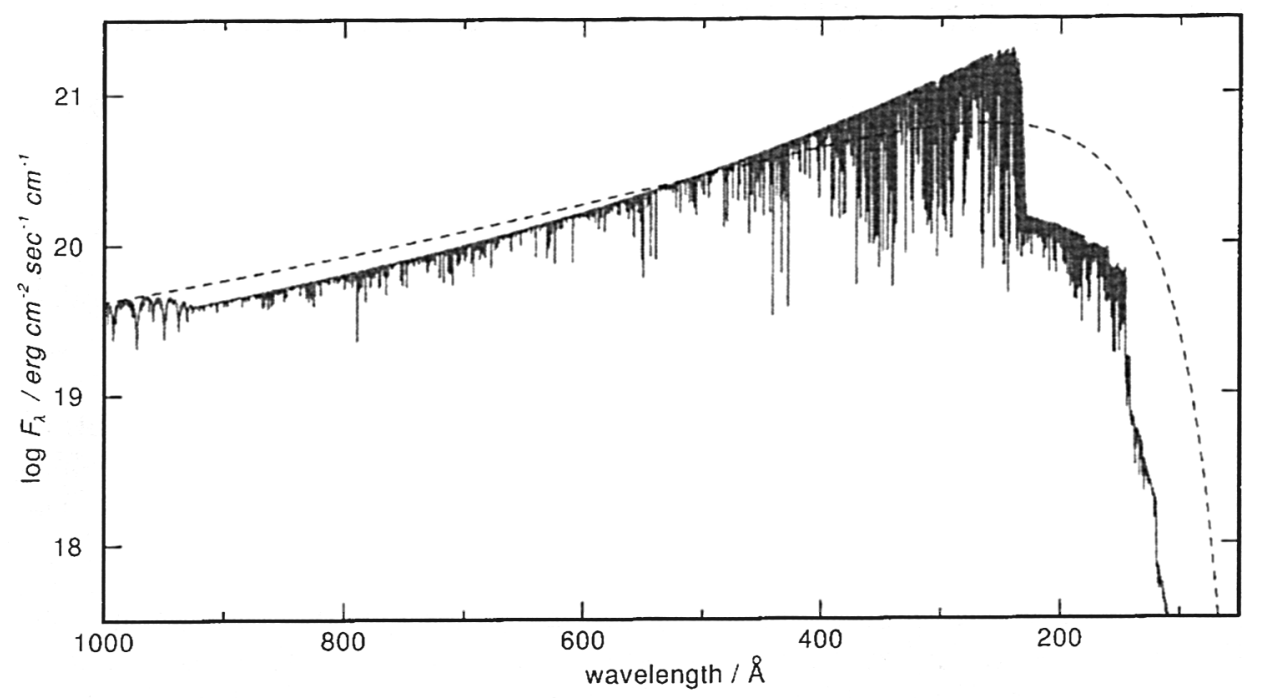

Figure 1. Comparison of a synthetic NLTE-model atmosphere flux $\left(T_{\text {eff }}=110 \mathrm{kK}, \log g=7\right.$ (cgs), solar abundances, elements $\mathrm{H}-\mathrm{Ni}$ ) with a black body flux (same temperature)

method (Cross-Section Creation Package CSC, Deetjen 1999, http://astro.unituebingen.de/ $\sim$ deetjen/csc.html).

Impacts of the light metals $\mathrm{Li}-\mathrm{Ca}$ are investigated by Rauch (1997). The influence of the iron-group elements is shown by Dreizler \& Werner (1993) and Deetjen et al. (1999).

A grid of $\mathrm{H}-\mathrm{Ca}\left(T_{\mathrm{e} f f}=50-1000 \mathrm{kK}, \log g=5-9\right.$ (cgs), solar and halo abundances) and $\mathrm{H}-\mathrm{Ni}\left(T_{\text {eff }}\right.$ up to $190 \mathrm{kK}$ ) model atmosphere fluxes is available at http://astro.uni-tuebingen.de/ $\sim$ rauch.

\section{References}

Armsdorfer B., Kimeswenger S., Rauch T. 2002, RevMexAA in press

Deetjen J.L. 1999, diploma thesis, University Tübingen

Deetjen J.L., Dreizler S., Rauch T., Werner K. 1999, in: White Dwarfs, ed. J.-E.Solheim \& E.G. Meištas, The ASP Conference Series Vol. 169, p. 475

Dreizler S., Werner K. 1993, A\&A 278, 199

Kurucz R.L. 1996, IAU Symp. 176, Kluwer, Dordrecht, p. 52

Rauch T. 1997, A\&A 320, 237

Seaton M.J., Yu Yan, Mihalas D., Pradhan A.K. 1994, MNRAS 266, 805

Werner K. 1986 A\&A 161, 177

Werner K., Dreizler S. 1999, Journal of Comp. and Appl. Mathematics 109, 65

Acknowledgments. This research was supported by the DLR under grant 50 OR 97055. 